\title{
ACHIEVING DATA ACCURACY BY SINR-BER MODELING IN MANETS
}

\author{
Mohammed Jawaharin Basha ${ }^{1}$, Shah Aqueel Ahmed ${ }^{2}$ \\ PhD- Research Scholar (ECE)-PP ECE.0088, Rayalaseema University, KURNOOL, A.P., India \\ jawaharinbasha@gmail.com \\ Professor of ECE, \& Principal, MMANTC Malegaon \\ informaqueel@gmail.com
}

\begin{abstract}
Wireless Ad hoc networks are an infrastructure less network with consists of wireless heterogeneous nodes, communicate one another through radio communication medium without central coordinator. Application of this network includes disaster relief, military and law enforcement. One of the challenging issues in MANETs is network scalability, which is greatly affected by interference. In literature different mechanisms developed to enhance the network scalability by network layer by considering per node power monitoring and link status monitoring. However, including these monitoring mechanisms, one of another considerable parameter is data accuracy. In this work we are proposes a BER-SINR model for MANETs to accurately detect the error coding for real time variable communication scenarios.
\end{abstract}

Keywords: MNETs, Data Accuracy, Network Capacity, BER-SINR, MAC Layer, $802.11 \mathrm{~b}$ $* * *$

\section{INTRODUCTION}

In our previous work we extend the network capacity by network layer protocols [1,2], while abstracting the other layers. However in order to enhance the network capacity another important factor is data accuracy and is achieved by medium access control layer. Thus in this work we initially examine the existing MAC protocol with respect to transmitter interference, signal transmission and reception. Moreover we enhance the existing model with SINR-BER $[7,8]$ extensions. Then we examine the how this extension enhance the network performance and used for capacity of network. We analyze this effect under different scenario under mobile ad hoc networks. Existing $802.11 \mathrm{~b}$ standard specifies three different types of PHY layer, which includes Frequency Hopping and Direct Sequence Spread Spectrum and infrared. Practically most popular PHY available type is Direct Sequence Spread Spectrum and operates in $2.4 \mathrm{GHz}$ ISM band with different modulation techniques for different data rates.

PHY layer enter into transmit state in order to transmit data, and if channel is free transmission of PPDU is initiated [9]

In mobile ad hoc networks, every mobile node has one or more network interface for communication with other nodes by making single physical channel, and calculates the propagation delay for communication and schedule the communication. At the receiving node computed the receiving power of packet by using below equation

$P_{r}=P_{t} G(d)$.
Where $P_{t}$, is transmitting power and $G(d)$ is link gain between source to destination with distance 'd'. And $G(d)$ is computed by the below equation for free space model

$G(d)=G_{t} G_{r} \lambda^{2} /(4 \pi d)^{2} L$

Or if $\mathrm{t}=\mathrm{it}$ is two ray ground model then $G(d)$ is calculated by below equation

$G(d)=G_{t} G_{r}\left(h_{t}{ }^{2} h_{r}{ }^{2}\right) /(d)^{4} L$ $-3$

Where $G_{t}, G_{r}$ are the gain of transmitter and receiver antennas and its default value is 1 , and $\mathrm{L}$ is the path loss \& $h_{t}, h_{r}$ are the heights transmitter and receiver antennas and default value is $1.5 \mathrm{~m}$.

Receiving power of packets is compared with two different parameters i.e, CST (carrier sense threshold) \& RXT (Receiver threshold).

If the receiving power of packet below the threshold value (CST) then packet is dropped by considering it as noise. Transmitting node does not initiate the transmission of new packet, if it senses the signal with power higher than CST threshold. Whereas RXT is used for the correct reception of packet. If received packet power is above the CST threshold but below the RXT, then packet is marked as error before processing to MAC layer, where packet is detected at destination but not successfully received. Or else packet is simply forwarded to MAC layer without any error. MAC layer of Destination contains one of either three states. 
1. MAC layer is ready to decode the packet, when it is in idle state

2. MAC layer is decoding the packet, when it is in receiving state

3. During decoding packet is collide, then MAC layer enter into Collision state.

A packet comes to MAC layer with idle state, then MAC layer switch to receive state and process the packet with computing transmission time. If MAC layer not in idle state, then receiving packet power level is lower than the current processing packet power level then arriving packet discarded and destination continues its current packet decoding, if it is not the case then collision occurs between arriving packet and current processing packet and then both the packets are dropped. Now the MAC layer in its collision state until both the packet completes its transmission, which prevents the sender to send new packet up to completion of collide packets transmission. This behavior work with CSMA/CA, and removes new transmission until the packet in a medium with receiving power greater than CST and also prevent the destination to listen new packet up to collision packets reception completed.

From above explanation it is clear that, decoding of packets carried out successfully until the received packet power level is enough to overcome the interference from other transmission and noise. This model work effectively by the process of every uncast packet follows the exchange of RTS/CTS control messages listen by most of the nodes present in a network. In this situation number of simultaneous interfering transmission is small and probability of packet reception is well achieved by comparing with single packet comparison. However, this model is not suited for a situation where the presence of multiple interfering transmission. Thus in our proposed work we enhancing the model to support simultaneous multiple interference.

\section{PROPOSED SYSTEM}

Probability of bit error is a degrading function of signal to noise ratio for single user adaptive white Gaussian noise link. In network with multiple user interference, it is necessary to calculate the communication link quality by adding signal to noise ratio with signal to interference (SINR). In order to calculate SINR, for a communication link with source let ' $i$ ', with transmitting power ' $P_{i}{ }^{\prime}$, and destination let ' $j$ ', . Power gain for link between source ' $i$, , to destination ' $j$ ', and receiving power ' $P_{j}$ '. Then SNIR for this link is calculated by below equation

$\gamma_{i}=\frac{G_{i i} P_{i}}{\sum_{j \neq i} \theta_{i j} G_{i j} P_{j}+\eta}$

Above equation denotes the in band receiver noise power level and denoted by $\gamma_{i}{ }^{\prime}$, this calculation includes thermal noise figure and receiver noise figure. Moreover, interference factor ' $\mu_{i j}$ ', denotes the fraction of source $i$ 's received signal power project up on the destination node $j$ 's signal space. Interference factor depends on number of factors such as modulation format, spreading code and data rates of source. Through analytical model, it conclude that ' $\mu_{i j}$ ' also depends on synchronous/asynchronous of the source transmission and hardware design implementation such as how many bits are used in anlog to digital conversion. If destination node using the filtering technique, which will cause the decrement I value of ' $\mu_{i j}{ }^{\prime}$. Moreover, ' $\mu_{i j}$ ' (interference factor) can be model for interfering signal which overlaps the frequency spectrum of node.

$i$. For a link $i$ transmitting at data rate $R i \mathrm{~b} / \mathrm{s}$, a common model is to assume that $\mu_{i j}=\mu i=R i=W$, corresponding to the reciprocal of the processing gain $N i=W=R i$.

In the context of 802.11 systems [3][4], the appropriate value of $\mu$ has received little attention, precisely because the carrier sense and request / CTS mechanisms have been designed to include all but very weak interfering transmissions. The fundamental change to the physical layer model is that in the receive state, a receiver node $i$ will track its SINR and the Fluctuations in the SINR will determine whether a packet is received correctly.

\section{PACKET RECEPTION}

In this work (model) we considered the physical model for reception of packet with excising IEEE 802.11 [3, 4] mechanism in the mobile ad hoc networks [5,6]. When the destination in idle state and ready to receive the packets and its received power level is greater than CTS control packet, then the medium access control layer enter into the receiving mode \& waits in this mode until the successful packet communication to be completed. This model is correlates with physical condition that a destination node gets the information such as length of packet, data rates from Packet header and this information is utilized to learn how many bits destination can decode. Destination node then checks the bit error with the help of CRC, after decoding the bits, if there is no error in packet reception then packets are forwarded to upper layer of protocol stack.

However, the probability of successful packet reception without error at destination is depends on destination SINR, which vary packet by packet. The further process of our method is to translate the SINR into packet error probability. This method of translation is set the factors such as modulation rate and error control coding. Translation is nothing but as a threshold value, correct packet reception only happen when the value of SINR above the threshold value during the packet interval. Moreover, packet error probability is an arbitrary function of SINR and is achieved by analytical model to physical layer .

BER based mechanism is used for a network with un coded packets, where detector makes the hard decision about every transmitted bit. This mechanism

Probabilistically decides whether every bit present in a packet successfully received by destination or not depends on destination SINR during the reception of bits. Each node stores the received signal power $P_{\text {total }}$ from multiple 
transmissions from different nodes and is reactively updated specific time interval. Implementation of SINR tracking is done by received power tracking, which is explained as follows

1. Whenever new packet received, extend the total received power $P_{\text {total }}$ by received power of that arriving packet

2. After completion of packet transmission, decrement the total power $P_{\text {total }}$ by received power of that packet

If a node gets a receiving packet with power $P_{r}$ and it contains the total power $P_{\text {total }}$, then nodes SINR is calculated as follows

$\gamma=\frac{P_{r}}{\theta\left[P_{\text {total }}-P_{r}\right]+\eta}$

The purpose of decoding packets, a constant SINR is developed such a way that

Segments of serial consecutive of received bits are formed. In BER depended model of packet reception is decide whether every received packet has error as a function of SINR in segment. Depends on this segment, reception of packets are defined as follows

1. Available segment, determine the bit error rate by utilizing table of BER-SINR (pre-computed).

2. If segment contains number of bits equal to $n$, then probability is calculated as follows

$P_{C}=\left(1-P_{e}\right)^{n}$

Such a way that all the bits available in segment correctly decoded. A uniform random variable value between $0 \& 1$ determine the estimated probability, if this value above the $P_{C}$, then segment contains error. If any decoding error occurs then discard the packet or else receive packet successfully .However, existing MAC standard does not use coding technique. Thus a segment contains single error, and then the packet could fail in CRC. Transmitted packet is lost either by collision or decoding error. However, novel concept of SINR tracking provides the correct packet decoding probability depends on all simultaneous interfering transmissions. After successful completion of decoding packet at destination, destination enters into the idle state, and it indicates that destination is ready to accept the packets. If any node enters into collision state, then node will remain in its state until all colliding packets transmitted.

\section{PROPOSED WORK}

A BER-SINR look up table is developed for packet reception. Error calculated for BER communication system is given by

$P_{e}=\frac{1}{2} e^{-\gamma b}$

Where $\gamma b$ is the SINR per bit. Every node present in a communication calculates this value and compares this value to its reference value stored in table for estimation.
The algorithm developed running per node is as presented below,

1. If transmitting power limit is in synchronization with receiving power, then interference observed by indicating the power fluctuation in receiving packet

2. Using obtained fluctuations SINR is calculated

3. Then calculated SINR mapped with estimated BER, and also computer the received packet BER

4. Then BER compared with estimated BER, it it is small then packet is received else packet rejected as noise.

Thus, Proposed BER-SINR model used for MANETs to achieve error coding for real time variable traffic scenarios. Evaluation is carried out for BER over variable SINR with respect to different data rates and shown in below figures2

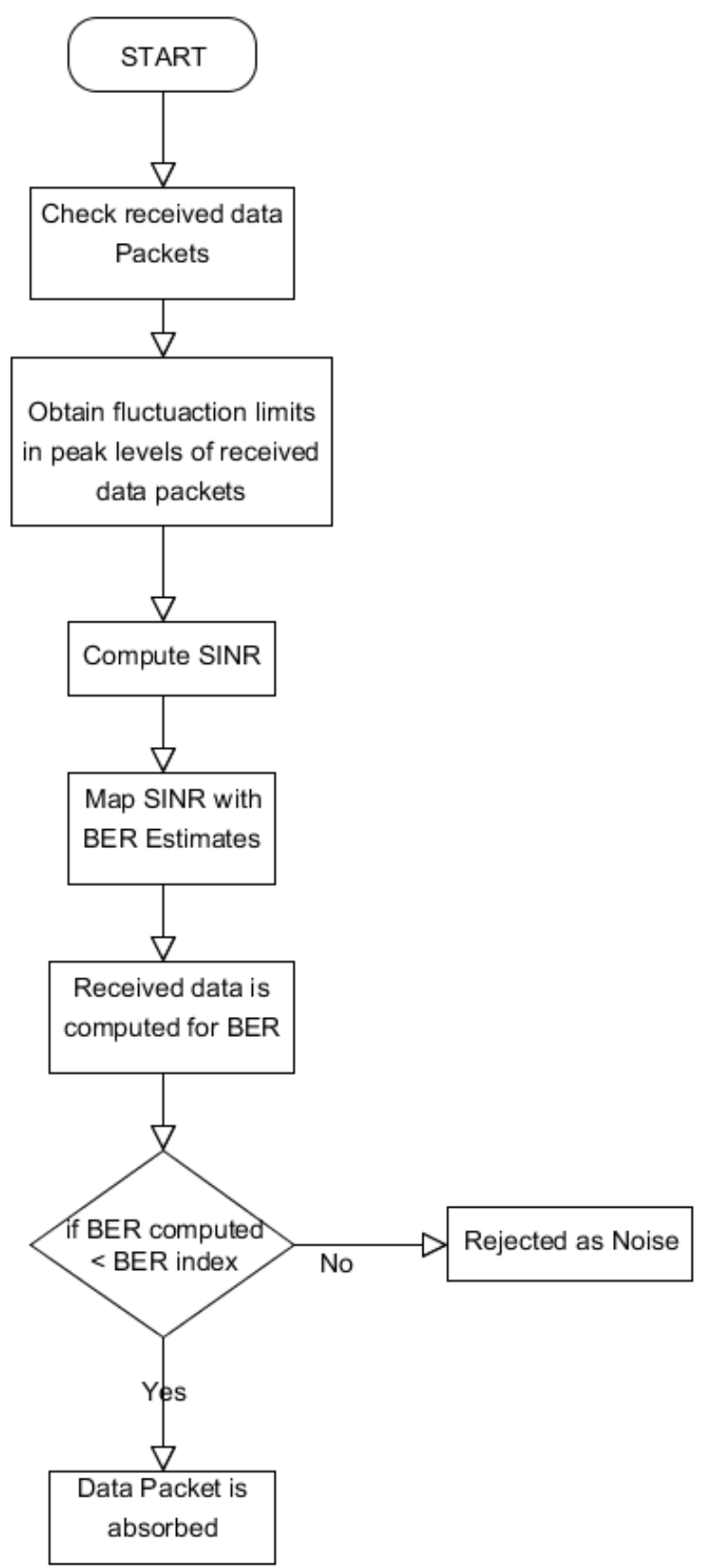

Figure 1: Flow chart of SINR-BER modeling 


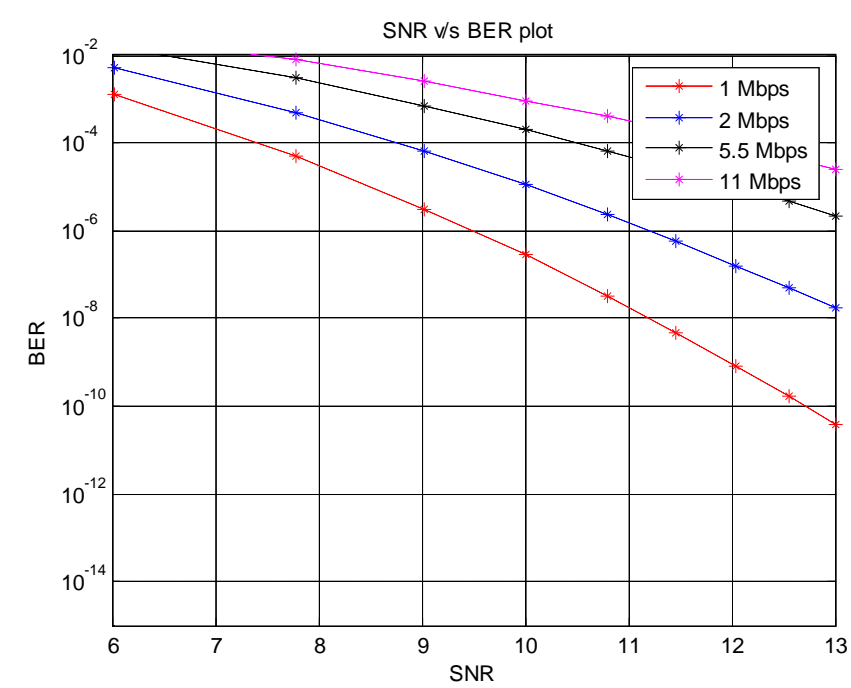

Figure 2: SNR v/s BER at variable data rate

Table 1: Observed BER over variable SNR for the developed wireless system

\begin{tabular}{|c|c|c|c|c|}
\hline \multirow{2}{*}{ SNR } & \multicolumn{4}{|c|}{ BER } \\
\cline { 2 - 5 } & 1 MBPS & 2 MBPS & 5.5 MBPS & 11 MBPS \\
\hline 6 & $10^{\wedge}-3$ & $10^{\wedge}-2.5$ & $10^{\wedge}-2$ & $10^{\wedge}-2$ \\
\hline 7.8 & $10^{\wedge}-5.6$ & $10^{\wedge}-3.8$ & $10^{\wedge}-3$ & $10^{\wedge}-2$ \\
\hline 9 & $10^{\wedge}-5.7$ & $10^{\wedge}-4$ & $10^{\wedge}-3.8$ & $10^{\wedge}-3.3$ \\
\hline 10 & $10^{\wedge}-7$ & $10^{\wedge}-5$ & $10^{\wedge}-4.2$ & $10^{\wedge}-3.5$ \\
\hline 10.8 & $10^{\wedge}-7.6$ & $10^{\wedge}-5.8$ & $10^{\wedge}-4.5$ & $10^{\wedge}-3.6$ \\
\hline 11.2 & $10^{\wedge}-8.5$ & $10^{\wedge}-7.8$ & $10^{\wedge}-4.6$ & $10^{\wedge}-4.0$ \\
\hline 12 & $10^{\wedge}-9$ & $10^{\wedge}-7.9$ & $10^{\wedge}-5.0$ & $10^{\wedge}-4.2$ \\
\hline 12.5 & $10^{\wedge}-9.8$ & $10^{\wedge}-7.5$ & $10^{\wedge}-5.2$ & $10^{\wedge}-4.6$ \\
\hline 13 & $10^{\wedge}-10.8$ & $10^{\wedge}-7.6$ & $10^{\wedge}-5.5$ & $10^{\wedge}-5$ \\
\hline
\end{tabular}

\section{CONCLUSION}

This work Proposes a BER-SINR model used for MANETs to achieve error coding for real time variable traffic scenarios to enhance the network performance and thereby enhancing the network capacity. Existing works enhances the network capacity by considering network layer protocols with monitoring mechanisms. Moreover, another network capacity enhancement metric for MANETs is data accuracy, which improved in this work.

\section{REFERENCES}

[1] Basha, Mohammed Jawaharin, and Shah Aqueel Ahmed. "REACTIVE POWER MONITOR ALGORITHM TO ENHANCE THE NETWORK CAPACITY." IJITR 5, no. 1 (2017): 5487-5491.

[2] Mohammed Jawaharin Basha Dr. Shah Aqueel Ahmed "REACTIVE LINK INTERFERENCE MONITORING TO IMPROVE NETWORK CAPACITY IN MANETS" IJEAST vol 2 issue 3 2017:55-59

[3] Poularakis, Konstantinos, et al. "Mobile Data Offloading Through Caching in Residential 802.11 Wireless Networks." IEEE Transactions on Network and Service Management 13.1 (2016): 71-84.
[4] Huang, Yangchao, et al. "Synchronized contention windows-based backoff algorithm in IEEE 802.11 wireless networks." Computer, Information and Telecommunication Systems (CITS), 2016 International Conference on. IEEE, 2016.

[5] Mohammad, Arshad Ahmad Khan, Ali Mirza, and Srikanth Vemuru. "Analytical Model for Evaluating the Bottleneck Node in MANETs." Indian Journal of Science and Technology 9.31 (2016).

[6] Mohammad, Arshad Ahmad Khan, Ali Mirza, and Srikanth Vemuru. "Cluster based mutual authenticated key agreement based on chaotic maps for mobile ad hoc networks." Indian Journal of Science and Technology 9.26 (2016).

[7] Schubert, Martin, and Holger Boche. "Solution of the multiuser downlink beamforming problem with individual SINR constraints." IEEE Transactions on Vehicular Technology 53.1 (2004): 18-28.

[8] Wang, Lu, Xiaoke Qi, and Kaishun Wu. "Embracing adjacent channel interference in next generation Wi-Fi networks." Communications (ICC), 2016 IEEE International Conference on. IEEE, 2016.

[9] Akyol, Umut. Effects of Physical Layer Models on Wireless Network Simulations. Diss. Rutgers, The State University of New Jersey, 2005. 